\title{
El uso de herramientas de toma de decisiones compartidas para el tamizaje de cáncer de mama aumentaría la negativa de las mujeres a realizarse una mamografía
}

\author{
Use of patient decision aids for breast cancer screening would increase women's refusal to have a mammogram
}

\author{
Comentado de: \\ IVlev I, et al. Journal of General Internal Medicine. 2017; 32 (7): \\ 803-812. PMID: 28289963 1
}

\section{Objetivos}

Evaluar el efecto que tiene el uso de herramientas de toma de decisiones compartidas (HTDC) a la hora de decidir iniciar o discontinuar el tamizaje con mamografía, en los grupos etarios donde la toma de decisiones compartidas está recomendada.

\section{Métodos de búsqueda}

La búsqueda se realizó en las siguientes bases de datos: Medline, Scopus, Cochrane, PsycINFO, Health and Psychosocial Instruments, Health Technology Assessment y PsycArticles, hasta agosto de 2016.

\section{Selección de estudios}

Se buscaron estudios primarios que incluyeran mujeres en el rango etario de 38 a 50 años (recomendación de toma de decisiones compartidas para decidir si inician tamizaje) y entre 68 y 89 años (recomendación de toma de decisiones compartidas para decidir si discontinuan tamizaje), sin diagnóstico previo de cáncer de mama, cuya intervención fuese la utilización de una HTDC. Se seleccionaron ensayos clínicos aleatorizados (ECA), ensayos clínicos no aleatorizados, cohortes, casos y controles y estudios de antes y después.

Los estudios elegibles fueron revisados por dos investigadores. El protocolo de esta revisión sistemática fue registrado en PROSPERO.

\section{Extracción de datos}

Se extrajeron los siguientes datos: edad de las participantes, riesgo basal o aumentado de cáncer de mama, tipo de intervención de cada estudio, y decisión final tomada por la paciente: seguras de querer participar del tamizaje, seguras de no querer participar, o inseguras al respecto.

\section{Resultados principales}

Se eligieron seis artículos: tres ensayos clínicos (ECA), con un total de 2.025 mujeres aleatorizadas, y tres estudios de antes y después, con 171 participantes en total.

Se combinaron los resultados de los tres ECA para realizar un meta-análisis, con modelo de efectos aleatorios. Se tuvo en cuenta la heterogeneidad y se calculó el I ${ }^{2}$. Se calculó el riesgo relativo (RR) y el número necesario a tratar (NNT).

De un total de 885 mujeres pertenecientes al grupo de intervención, 121 decidieron no iniciar o discontinuar el tamizaje con mamografía, versus 103/984 mujeres en el grupo de control. Esto implica que menos mujeres se realizarían la mamografía luego de utilizar una HTDC, con un RR de 1,48 (Intervalo de confianza [IC] $95 \%: 1,04$ a 2,13 ) y un NNT de 20.

En el grupo etario de 38 a 50 años la diferencia fue aún mayor (RR: 1,77; IC $95 \%$ : 1,34 a 2,34; NNT: 12), mientras que en el subgrupo de 69 a 71 años no se encontraron diferencias significativas.
Como resultados secundarios se encontraron diferencias a favor en mejoras en el conocimiento de las mujeres (tres estudios), toma de decisión informada (dos estudios), seguridad y conocimiento a la hora de tomar la decisión y menos incertidumbre al momento de decidir, en las mujeres que habían recibido la intervención.

Los estudios antes y después eran de baja calidad, y no aportaron diferencias en ninguno de los desenlaces o subgrupos evaluados.

\section{Conclusiones}

Los resultados de este estudio muestran que las mujeres que utilizan HTDC para tamizaje de cáncer de mama son menos proclives a realizarse mamografías.

\section{Comentario}

Esta es la primer revisión sistemática realizada para evaluar el impacto de las HTDC en el tamizaje de cáncer de mama. Se focaliza en la decisión final tomada por las mujeres de participar del tamizaje o no, y pareciera ser que las mujeres que son expuestas a mayor información sobre el tema deciden participar del tamizaje en menor medida.

Sin embargo, este estudio nos deja con varios interrogantes. Como punto inicial, se eligieron mujeres de un rango etario específico, dejando de lado a las mujeres entre 50 y 70 años, para las que tampoco hay estudios que hayan demostrado que el rastreo con mamografía mejore los índices de mortalidad, y con resultados nada despreciables con respecto a sobrediagnóstico y falsos positivos, por lo que cabría preguntarse si estas mujeres no son también candidatas a utilizar HTDC.

También es llamativa la elección del desenlace principal. El objetivo del uso de las herramientas es ayudar a las pacientes a tomar una decisión informada, con información objetiva disponible, sopesando los pros y los contras de cada opción y basándose en sus propios valores y preferencias ${ }^{2}$. Con este concepto, nuestros desenlaces de interés deberían ser la toma de decisión informada y el conocimiento adquirido por las mujeres, mientras que la opción final que tome cada paciente quedaría relegada a un segundo plano.

Una revisión sistemática posterior ${ }^{3}$ evaluó el efecto del uso de HTDC en mujeres de 50 años que debían enfrentarse a la decisión de iniciar o no tamizaje con mamografía. Sus desenlaces principales fueron la toma de decisión informada, el conflicto para tomar la decisión y el conocimiento respecto al tema en cuanto a mortalidad, falsos positivos y sobrediagnóstico. Incluyeron tres ensayos clínicos aleatorizados y un estudio de antes y después. El uso de las HTDC aumentó la proporción de mujeres que lograron tomar la decisión informada en 14\% (IC 95\%: 2 a 27), y la proporción de mujeres con conocimiento adecuado en $12 \%$ (IC $95 \% 7$ a 16). Secundariamente, se observó que menos mujeres decidían participar del tamizaje.

Por otro lado, cabe destacar que los estudios incluidos fueron realizados en Australia y los EE.UU. con herramientas diseñadas específicamente para esas poblaciones, y con datos y gráficos 
algo disímiles entre sí. Por estas razones, parece cuestionable la agrupación de estos estudios para realizar un meta-análisis, y más aún, intentar extrapolar estos resultados a poblaciones de otras características.

\section{Conclusiones de la comentadora}

La evidencia sobre la utilidad de las herramientas de toma de decisiones compartidas para el tamizaje del cáncer mamario continúa siendo escasa, y serán necesarios nuevos estudios realizados en poblaciones de diferentes países para poder sacar mejores conclusiones.

Josefina Chiodi [ Servicio de Medicina Familiar y Comunitaria, Hospital Italiano de Buenos Aires. josefina.chiodi@hospitalitaliano.org.ar ]

Chiodi J. El uso de herramientas de toma de decisiones compartidas para el tamizaje de cáncer de mama aumentaría la negativa de las mujeres a realizarse una mamografía. Evid Actual Pract Ambul. 2020;23(1):e002026. Comentado de: Ivlev I, et al. Use of patient decision aids increased younger women's reluctance to begin screening mammography: a systematic review and meta-analysis. J Gen Intern Med. 2017;32(7):803-812. PMID: 282899639

\section{Referencias}

1. Ivlev I, Hickman EN, McDonagh MS, et al. Use of patient decision aids increased younger women's reluctance to begin screening mammography: a systematic review and meta-analysis. Journal of General Internal Medicine. 2017;32(7):803-812.

2. Weston WW. Informed and shared decision-making: the crux of patient-centered care. CMAJ: Canadian Medical Association journal. 2001;165(4):438-439.

3. Martínez-Alonso M, Carles-Lavila M, Pérez-Lacasta MJ, et al. Assessment of the effects of decision aids about breast cancer screening: a systematic review and meta-analysis. BMJ Open. 2017;7(10):e016894. 